\section{The Total Release Method for FIB In-Situ TEM Sample Preparation}

\section{T.M. Moore}

Omniprobe, Inc., Dallas, TX

\section{moore@omniprobe.com}

In 1965, Gordon Moore forecast that the microprocessor industry would continually scale to smaller feature sizes and the number of transistors would double every 18 months. Scaling below the $100 \mathrm{~nm}$ node, combined with the implementation of copper and low dielectric constant insulators to increase the processor speed, has produced the situation in which SEM inspection no longer offers suitable resolution to image key artifacts and structures. The transmission electron microscope (TEM), once considered more of a development tool, is now in the forefront for process control and failure analysis, especially for measurements such as the thickness of semiconductor device non-planar barrier and seed layers. The use of focused ion beam (FIB) microscopes has become the method of choice for site-specific TEM sample preparation. Originally, the FIB was used as a final thinning step for mechanically prepared ribbons of semiconductor material adhered to modified TEM grids, known as the "H-Bar" method. More recently, the method for performing the entire TEM sample preparation process within the FIB is known as "in-situ lift-out" and is based on the use of a chamber-mounted nanomanipulator and beam-induced material deposition. [1-5] The use of the FIB offers advantages over conventional mechanical TEM sample preparation. The dual-beam FIB offers the ability to locate the lift-out site with SEM resolution and then use the ion beam to excise the sample without sacrificing the wafer, followed by thinning the extracted sample to the thickness required for TEM inspection. This is especially attractive for $300 \mathrm{~mm}$ processing where the value of each wafer in the flow can exceed $\$ 100,000$. The risk to the quality and reliability of the process wafer due to gallium contamination from the ion beam is considered manageable.[6] In-situ lift-out also enables the return of the mostly abandoned practice of including informative test die on product wafers.

The Total Release method for in-situ lift-out is designed to maximize throughput of a TEM sample preparation process. [4-5] The method can be simplified into three successive steps (see Figure 1). The first is the excision of the lift-out sample using FIB milling and extraction of the sample from its trench with two rapid ion milling steps, or "cuts". The first cut is "U"-shaped and partially surrounds the target. The second is a straight cut that intersects the first cut beneath the target and produces a wedge-shaped sample. Then the probe is fixed to the released sample, typically with ionbeam metal deposition, and the sample is removed from the wafer by the nanomanipulator. The second step is the "holder-attach" step, during which the wedge is translated on the probe tip to the TEM sample holder (the lift-out grid). Then the sample is attached to the TEM holder (again, typically with ion beam-induced metal deposition) and later detached from the probe tip point using FIB milling. The third and final step is the thinning of the wedge into an electron-transparent thin section using FIB milling.

The use of a simple probe tip for lift-out in the FIB has throughput and efficiency advantages over alternative methods. For example, lift-out can also be accomplished by a method referred to as ex-situ lift-out, in which ion beam-thinned samples, still attached to the wafer, are removed from the FIB and then detached from the wafer with a statically charged glass needle. These samples are then permanently deposited onto the suspended areas on a polymer membrane-coated TEM grid. Ex-situ lift-out can be faster than the in-situ method, and it requires less time in the FIB. However, in-situ lift-out offers a much higher overall throughput through
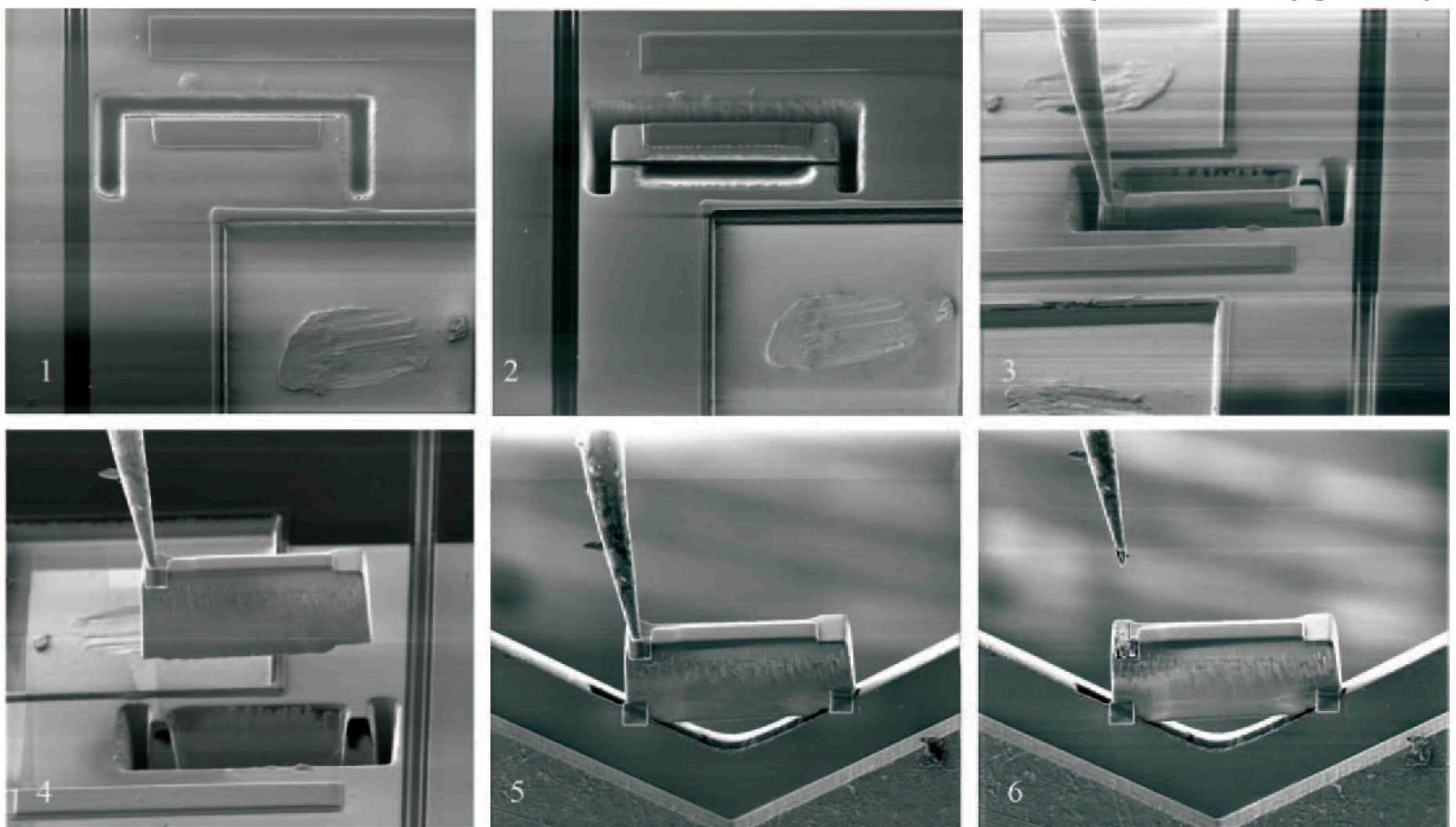

Fig.1. Typical steps of in-situ lift-out with the Total Release method. (1) first cut; (2) release cut; (3) tip attach; (4) extraction; (5) holder attach; and (6) tip separation. 


\section{Omniprobe Short-Gut th brings}

\section{HIHI THBOUHIPUT \\ to in-situ TEM sample preparation}

\section{In-Situ FIB Lift-Out}

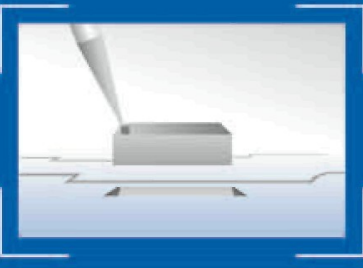

Streamlined in-situ

FIB lift-out with

Omniprobe's

patented Total

Release $^{\mathrm{TM}}$

technology

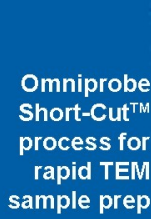

TEM sample produced using

Omniprobe Short-Cut ${ }^{\text {TM }}$ Tool

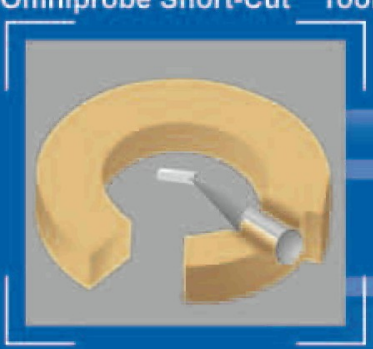

\section{Mechanical Conversion}

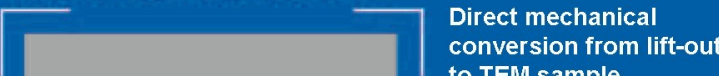

to TEM sample

TEM Sample Prep And Nano-Mechanical Testing

The AutoProbe ${ }^{T M} 200$ is a computer controlled in-situ multipurpose nanomanipulator system capable of

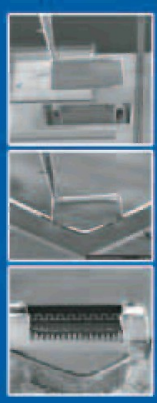

- Lift-out for TEM Sample Prep

- Electrical Measurements

- Nano-Mechanical Testing

Ideal For:

- IC Process Control and

Failure Analyses

- Topographic Surfaces

- Brittle or Soft Materials

- Phase Boundaries

- MEMS Components

C $\epsilon$ and UL certified

Patented Technology

AutoProhe'm 200

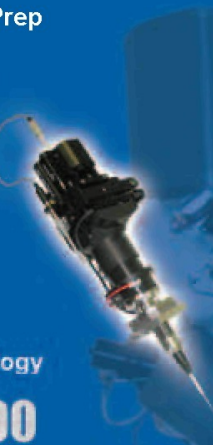

\section{Computer Controlled Multi-Gas Injection System}

The OmniGISTM is Omniprobe's new multiple gas injection system for the FIB or SEM. The OmniGIS ${ }^{T M}$ provides programmable control of three gas sources and an additional purge/carrier gas source.

- Programmable Process Flow

- 3 Independent Gas Sources

- User-Replaceable Crucibles

- Feedback Control

- Patent Pending

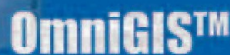

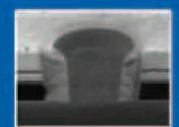

Multiple Gas Sources (Oxide Isolated Pt Via)
European Support: SEM-FIB Solutions +44 1993771203 tony.kendrick@sem-fib.com Oxford, United Kingdom 


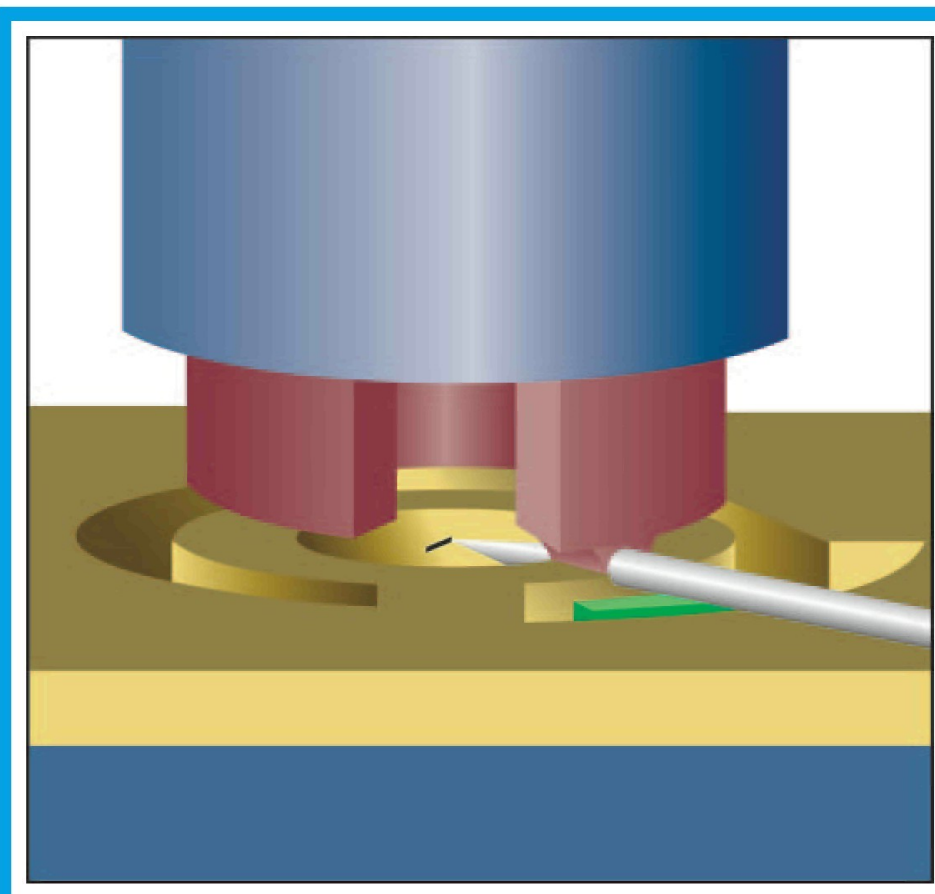

Fig.2. A schematic of the press for mechanical conversion, the TEM holder coupon and the probe tip with sample attached.

automation of the process within the FIB environment. In-situ lift-out also provides the ability to return the TEM sample to the FIB for additional thinning, with better protection of the delicate sample, better thermal and electrical grounding of the sample in the TEM, and cleaner samples. In another alternative approach, a mechanical device such as a MEMS gripper can be used to remove the excised sample in the FIB.[7] Compared to the use of a MEMS gripper to retrieve the sample, the probe tip approach offers a much lower consumable cost ( $<\$ 10 /$ tip vs $>\$ 500 /$ gripper), more reliable handling of the sample, much less ion milling to excise the sample, and better alignment of the sample on the lift-out grid for TEM inspection.

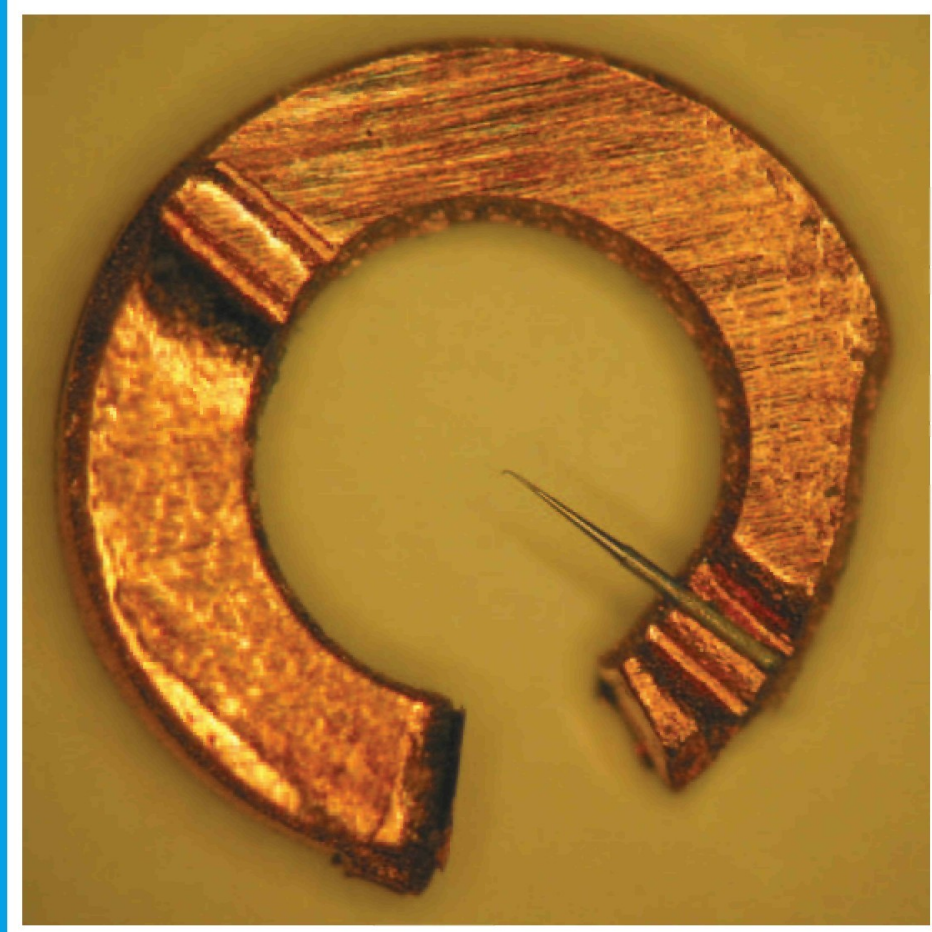

Fig.3. The finished 3mm TEM sample holder designed for top-side milling with one tip attached.
The Total Release method has several attractive features for a high-throughput TEM sample preparation process. It requires a minimum of ion milling to excise the sample and provides reliable and protected transport of the sample. It allows for instant and definitive indication that the sample release procedure has been completed successfully, thus saving time. If pressure is applied by the probe tip to the sample during the final milling procedure, a change in mechanical load measured by a sensitive strain gauge inside the probe shaft of the nanomanipulator can be used to indicate that the sample release procedure has been successfully completed. [8] This same strain gauge can be used to detect contact between the tip of the probe and the sample in an automated process. Such a detection method is independent of electrical continuity effects and functions efficiently with a fine probe tip on any type of sample surface. Since the probe tip is not attached to the sample prior to total release, a premature lift-out attempt can be avoided, and re-deposition in the initial stage of the lift-out process can be more easily cleared without having to detach the probe tip.

The throughput advantages of the Total Release method can be augmented by an improvement in sample handling that eliminates the need for the holder attach step. A significant portion of the total time is involved in completing this step. The relative amount of time involved depends on the amount of time required to mechanically isolate the lift-out sample from the initial bulk sample (materialdependent ion beam milling rate), and will vary between $30 \%$ to $60 \%$ of the total time for TEM sample preparation. However, direct mechanical conversion of the lift-out sample to the TEM sample holder eliminates the holder-attach step, and provides several key through-put and resource advantages (see Figures 2 and 3). [9] For example, the semiconductor wafer can be returned to the process flow immediately after lift-out. Thinning of the sample can be performed immediately, or later in an off-line FIB. The use of the off-line FIB for final thinning reduces the load on the critical in-line (clean room) FIB and is well-suited for an automated sample preparation procedure. This mechanical conversion process provides rapid, low cost and robust bonding of the probe tip point and the standard 3 mm TEM holder.

As more semiconductor products migrate to sub- $100 \mathrm{~nm}$ geometries, the need for low- cost, high-throughput and site-specific TEM sample preparation will increase in importance. The Total Release method for TEM sample preparation provides an effective solution, and offers excellent throughput advantages through process automation.

\section{References}

[1] L.A. Giannuzzi et al., "FIB Lift-Out Specimen Preparation Techniques", in Introduction to Focused Ion Beams: Instrumentation, Theory, Techniques, and Practice, L.A. Giannuzzi, F.A. Stevie (eds), Springer-Verlag New York, 201 (2004).

[2] T. Kamino et al., "A FIB Microsampling Technique and a Site Specific TEM Specimen Preparation Method", in Introduction to Focused Ion Beams: Instrumentation, Theory, Techniques, and Practice, L.A. Giannuzzi, F.A. Stevie (eds), Springer-Verlag New York, 229 (2004).

[3] T. Ohnishi et al., U.S. Patent 5270552.

[4] T.M. Moore et al., U.S. Patent 6420722.

[5] T.M. Moore, U.S. Patent 6570170.

[6] T.E. West, et al., "Inline Failure Analysis $n$ Productive Wafers with Dual Beam SEM/FIB Systems", Future Fab International, Montgomery Research (pubs), Volume 11 (2001).

[7] K. Tuck, et al., "FIB Prepared TEM Sample Lift-out Using MEMS Grippers", Proceedings Microscopy and Microanalysis 2004, Cambridge University Press, 1144 (2004).

[8] Omniprobe AutoSense ${ }^{\mathrm{sx}}$ probe shaft, patent pending.

[9] Omniprobe Short-Cut ${ }^{\text {max }}$ system, patent pending.

[10] The authors wish to thank to G. Amador, C.D. Hartfield and R. Kruger for their dedicated efforts. 


\section{INCADryCool}

Liquid

Nitrogen-Free

Detector

\section{No compromise on} analytical performance

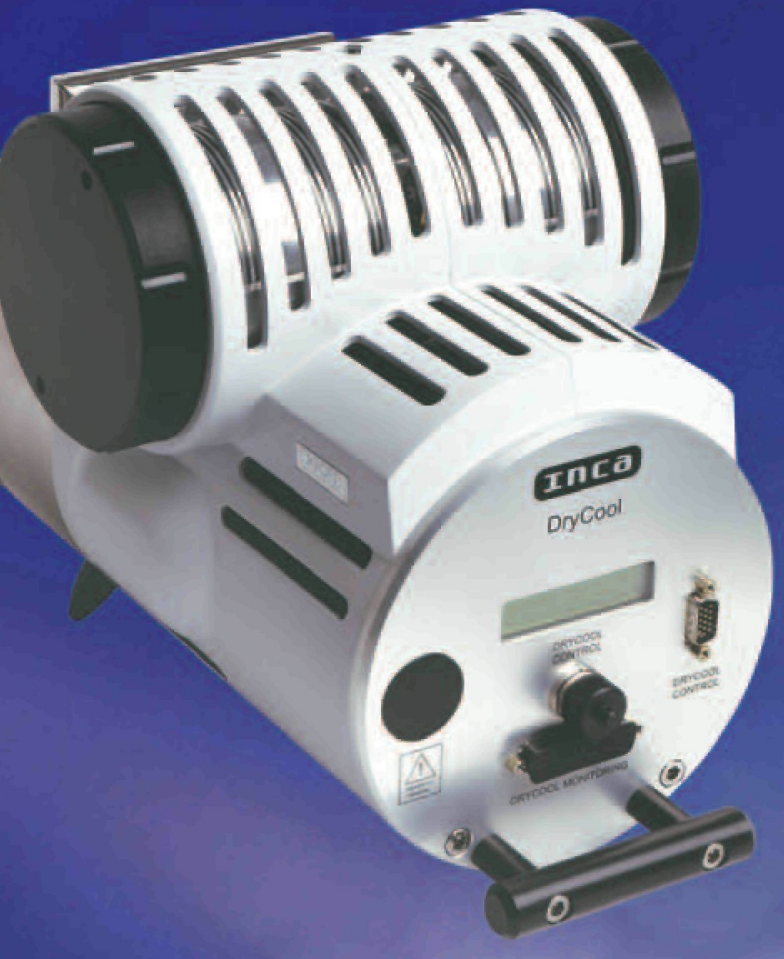

Convenience of $\mathrm{LN}_{2}$ free operation

Analyze the full elemental range of Be - Pu with confidence

- Suitable for all SEMs - at the highest resolution

Reliability of cooling technology developed for space applications

- Rapid delivery - no waiting!

Interested?

Find out more from:

Oxford Instruments USA

Concord, MA

Tel: +1 9783699933 Toll Free: +1 8004474717

Email: info@ma.oxinst.com

www.oxford-instruments.com 